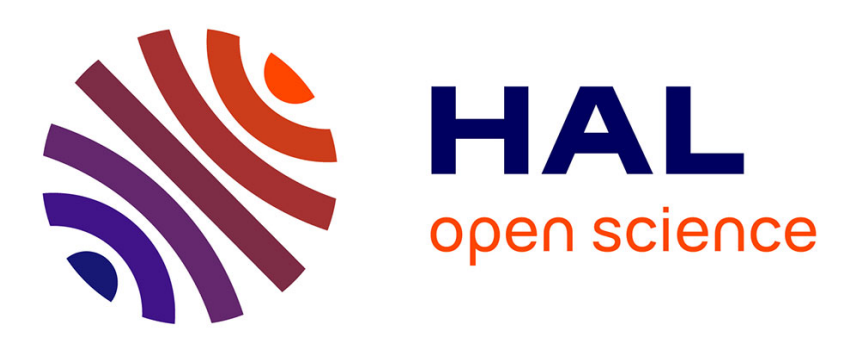

\title{
On the stability of nonconservative elastic systems under mixed perturbations
}

Noël Challamel, François Nicot, Jean Lerbet, Félix Darve

\section{To cite this version:}

Noël Challamel, François Nicot, Jean Lerbet, Félix Darve. On the stability of nonconservative elastic systems under mixed perturbations. European Journal of Environmental and Civil Engineering, 2009, 13 (3), pp.347-367. 10.1080/19648189.2009.9693112 . hal-00647352

\section{HAL Id: hal-00647352 \\ https://hal.science/hal-00647352}

Submitted on 26 Dec 2019

HAL is a multi-disciplinary open access archive for the deposit and dissemination of scientific research documents, whether they are published or not. The documents may come from teaching and research institutions in France or abroad, or from public or private research centers.
L'archive ouverte pluridisciplinaire HAL, est destinée au dépôt et à la diffusion de documents scientifiques de niveau recherche, publiés ou non, émanant des établissements d'enseignement et de recherche français ou étrangers, des laboratoires publics ou privés. 


\title{
On the stability of non-conservative elastic systems under mixed perturbations
}

\author{
Noël Challamel ${ }^{*}$ - François Nicot $* *$ - Jean Lerbet ${ }^{* * * *}$ \\ Félix Darve *****
}

* LGCGM - INSA de Rennes - Université européenne de Bretagne 20, avenue des Buttes de Coësmes, F-35043 Rennes cedex

noel.challamel@insa-rennes.fr

** CEMAGREF, Unité de Recherche Erosion Torrentielle - Neige et Avalanches

2, rue de la papeterie, F-38042 Saint-Martin-d'Heres cedex

Francois.nicot@grenoble.cemagref.fr

*** Université d'Evry, Val-d'Essonne, UFR Sciences et Technologie

40, rue du Pelvoux CE 1455, F-91020 Evry-Courcouronnes cedex

Jean.lerbet@ibisc.univ-evry.fr

**** INPG, Laboratoire Sols, Solides, Structures

BP 53, 40, rue du Pelvoux CE 1455, F-38041 Grenoble cedex 9

RÉSUMÉ. Cet article étudie l'influence du mode de chargement sur la stabilité de systèmes élastiques discrets non conservatifs. La stabilité du système contraint est comparée à celle du système libre, par l'introduction de multiplicateurs de Lagrange. L'approche est illustrée avec le pendule généralisé de Ziegler. Elle est ensuite appliquée à un modèle à deux degrés de liberté représentant un sol contraint par un chargement isochore. On montre que le chargement isochore affecte sensiblement la frontière de stabilité pour le problème conservatif et pour le problème non conservatif. En dehors des instabilités par flottement, le critère de travail du second-ordre constitue une borne inférieure de la frontière de stabilité du système libre ainsi que la frontière du domaine de stabilité du système sous chargements mixtes proportionnels en déplacement.

ABSTRACT. This paper shows that the loading mode strongly influences the stability of discrete nonconservative elastic systems. The stability of the constrained system is compared to the stability of the unconstrained system, through the incorporation of Lagrange multipliers. Initially, the approach is illustrated for a two-degrees-of-freedom generalized Ziegler's column. Then, it is applied to a two-degrees-of-freedom model representing a soil constrained with isochoric loading. The isochoric instability load is not necessarily greater than the instability load of the free problem. Excluding flutter instabilities, it is shown that the second-order work criterion is not only a lower bound of the stability boundary of the free system, but also the boundary of the stability domain, in presence of mixed perturbations based on proportional kinematic conditions.

MOTS-CLÉS : stabilité des structures élastiques, problèmes non conservatifs, perturbations mixtes, systèmes contraints, stabilité matérielle, chargement isochore.

KEYWORDS: stability of elastic structures, non-conservative problems, mixed perturbations, constraints, material stability, isochoric loading. 


\section{Introduction}

Stability of elastic structures is a branch of engineering that has been well developed since the 1960s, especially in presence of non-conservative loading (e.g. Bolotin, 1963; Ziegler, 1968). Most of these studies have investigated nonconservative loading such as circulatory loading. Another origin of nonconservativeness is the nonlinear elastic behaviour with a non-symmetric Hessian tensor (Green and Naghdi, 1971). Cauchy elasticity may typically belong to such class of non-consevative elastic material (see more recently (Rubin, 2009)). Such non-conservative constitutive elastic relations are sometimes used in soil mechanics, as analysed by Zytynski et al. (1978) or more recently by Houlsby et al. (2005). Such constitutive relations may be also encountered when modelling anisotropic damage in quasi-brittle materials with unilateral effects (Carol, Willam, 1996; Challamel et al., 2006a). Moreover, inelastic behaviour such as plasticity or damage may also reveal an asymmetric stiffness matrix for the relationship between the stress rate and the strain rate (incremental response, e.g. (Darve, Vardoulakis, 2005)). This asymmetric matrix property is typically observed for non-associated plasticity models (Imposimato, Nova, 1998; Darve, Laouafa, 2000; Darve et al., 2004; Nicot et $a l ., 2007$ ) or damage models based on Mazars positive strain (Challamel et al., 2006b). Moreover, the incompressible property of the flow rule is often employed when modelling ductile materials such as soil in undrained conditions, or metal. Therefore, it is crucial to rigorously investigate the effect of the incompressibility feature on the stability domain based on stability theory. This study also investigates polymer materials that may have the incompressibility property. However, in this paper, incompressibility is not understood as an intrinsic property of a material, but as a loading feature. We would employ the terminology isochoric loading to characterise such loading. The present study is restricted to elastic systems, since stability of inelastic media may reveal additional complexities (and in particular, the non-smooth character of the constitutive relation; see for instance Challamel, Pijaudier-Cabot, 2006c; Challamel, Gilles, 2007). Nevertheless, it is hoped that some of the conclusions of this study will be transposable to inelastic stability studies, based on the concept of Hill's linear comparison solid (for incrementally piece-wise linear rate-independent constitutive relations, see Nguyen, 2000; Chambon et al., 2004).

This paper uses a simple discrete elastic example to demonstrate the incompressibility effect on the stability boundary. The non-conservative nature of the problem may result from loading or from the constitutive law, and a single general criterion is derived for both problems. In other words, the treatment of the material's stability problem and the structural stability problem is merged into a general framework. It is shown that the incompressibility property strongly affects the stability boundary. A two-degrees-of-freedom buckling problem illustrates the new results. 


\section{Equations of the dynamics - some usual stability results}

\subsection{Lagrange's equations}

The Lagrange's equations (Ziegler, 1968) of a dynamical holonomic system could be written as:

$$
\frac{d}{d t} \frac{\partial T}{\partial \dot{q}_{k}}-\frac{\partial T}{\partial q_{k}}=Q_{k}\left(q_{k}, \dot{q}_{k}\right)
$$

where $T$ is the kinetic energy and $Q_{k}$ is the generalized forces. $\underline{q}$ is a state variable of dimension $n$. It is chosen to linearize the dynamics equations in the vicinity of an equilibrium position denoted by $\underline{q_{e}}$ such as:

$$
\underline{q}=\underline{q}_{e}+\underline{x}
$$

The linear differential system is then obtained for the perturbation equations:

$$
\underline{\underline{M}} \underline{\ddot{x}}+\underline{\underline{C}} \underline{\dot{x}}+\underline{\underline{K}} \underline{x}=\underline{0}
$$

where the matrix $\underline{\underline{K}}$ is generally a non-symmetric matrix. Matrix $\underline{\underline{M}}$ is assumed to be a definite positive matrix. The general properties of such a damped nonconservative system have been extensively studied since the 1960's. A detailed analysis of flutter and divergence bifurcation phenomena can be found in the recent paper of Kounadis (2007).

\subsection{Divergence criterion}

The stability concept used in this paper is stability in Lyapunov's sense, which expresses the property of uniform continuity of the perturbed solution to initial conditions. For the undamped system, the stability criteria are greatly simplified. In a conservative system, the matrix $\underline{\underline{K}}$ is symmetric. Stability of the equilibrium can be investigated by means of the Lagrange-Dirichlet criterion. The definite positiveness of the stiffness matrix $\underline{K}$ is easily checked from Sylvester's criterion (La Salle and Lefschetz, 1961). The loss of definite positiveness is reached when the determinant of one of the submatrices of Sylvester's criterion vanishes. The eigenvalues of the matrix $\underline{\underline{K}}$ are assumed to depend continuously on the loading parameters. The boundary between stability and instability is generally given by the singularity condition:

$$
\operatorname{det}(\underline{\underline{K}})=0
$$


In this undamped case, stability is associated with the stability in Lyapunov's sense, or simple stability. It is worth mentioning that the proof of stability is obtained by applying Lyapunov's direct method. In a non-conservative system, the matrix $K$ is no longer symmetric: stability may be lost by divergence or by flutter. Instability by divergence is typically observed in conservative systems, one of the most popular examples being Euler buckling. The loss of stability in which the structure is oscillating at the critical load is called the oscillatory instability, or the flutter phenomenon. This kind of instability has been known for years in the field of aeroelasticity, for the bending-torsional flutter of a cantilever wing for instance (e.g. Bolotin, 1963). For civil engineering applications, the behaviour of suspension bridges may also result from flutter, as in the well-known Tacoma bridge collapse in 1940 (Bažant and Cedolin, 2003; see more recently Plaut and Davis, 2007 or Plaut, 2008). The "static" stability criterion Equation [4] can be used again when stability is lost by divergence (Leipholz, 1987; Gajewski and Zyczkowski, 1988). As the symmetry of the matrix $K$ is no longer guaranteed for non-conservative systems, the criterion of definite positiveness of the stiffness matrix and the divergence criterion given by Equation [4] have to be strictly distinguished. Flutter instabilities can be detected with a procedure available in the literature (see Afolabi, 1995; Gallina, 2003).

\subsection{The definite positiveness of the stiffness matrix criterion}

The definite positiveness of the stiffness matrix criterion can be enunciated as:

$$
\underline{\underline{K}} \text { definite positive } \Rightarrow \text { stability }
$$

This criterion is also encountered in problems of inelastic evolutions and is sometimes called the second-order work criterion, or Hill's criterion (e.g. Nicot et al., 2007). The Equation [5] criterion is a sufficient condition of stability, in case of instability loss by divergence. The proof for elastic systems was rigorously given by Huseyin and Leipholz (1973). Absi and Lerbet (2004) also applied this criterion to Ziegler's pendulum (Ziegler, 1968). It is worth mentioning that the definite positiveness of $\underline{\underline{K}}$ is strictly equivalent to the definite positiveness of the symmetric part of $\underline{\underline{K}}$, denoted by $\underline{\underline{K}}^{S}$. Sylvester's criterion may also be applied to the symmetrical matrix $\underline{\underline{K}}^{S}$. The loss of positive definiteness is reached when the determinant of $\underline{\underline{K}}^{S}$ is vanishing. As a consequence, the boundary of the secondorder work criterion may be written as:

$$
\operatorname{det}\left(\underline{\underline{K}}^{S}\right)=0
$$

Both criteria, Equations [6] and [4], are merged for conservative systems. However, for non-conservative systems, the Equation [6] criterion gives a lower 
bound of the exact stability criterion in Equation [4], when instability prevails by divergence (see recently Lerbet et al., 2007). This theorem was shown for the first time, to our knowledge, by Huseyin and Leipholz (1973). The proof of this theorem can be based on a mathematical result given by Ostrowski and Taussky (1951):

$$
\underline{\underline{K}} \text { definite positive } \Rightarrow \operatorname{det}(\underline{\underline{K}}) \geq \operatorname{det}\left(\underline{\underline{K}} \underline{\underline{S}}^{S}\right)>0
$$

These inequalities are strictly verified for asymmetric matrix $\underline{\underline{K}}$. The results can be also shown using Bromwhich bounds, as noted by Willam and Iordache (2001). The theorem of Huseyin and Leipholz (1973) rigorously states that the second-order work criterion gives a lower bound of the exact stability criterion, when instability prevails by divergence or when instability by flutter appears for higher critical loads than that leading to divergence. However, the second-order work criterion no longer guarantees the stability (in Lyapunov's sense) when there is stability loss by flutter. On a two-degrees-of-freedom system (extension of Ziegler's column), Challamel (2005) showed that the second-order work criterion could be a lower bound or an upper bound of the exact stability criterion, depending on the structure of the mass matrix (instability arises by flutter for this system).

\section{Effect of additional constraints}

We would like to investigate the properties of such a dynamical system in presence of an additional constraint, given by:

$$
f(\underline{q})=0
$$

Such additional constraint could be suggested for instance to stabilize the system in structural mechanics. This additional constraint could be also associated to an isochoric condition in soil mechanics. Therefore, the new dynamical system is written as:

$$
\frac{d}{d t} \frac{\partial T}{\partial \dot{q}_{k}}-\frac{\partial T}{\partial q_{k}}=Q_{k}\left(q_{k}, \dot{q}_{k}\right)+\lambda \frac{\partial f}{\partial q_{k}}
$$

where $\lambda$ is a Lagrange multiplier associated to the holonomic constraint Equation [8]. It is assumed furthermore, that the equilibrium position of the free system is also an equilibrium position of the constrained system:

$$
f\left(\underline{q_{e}}\right)=0
$$

The dynamics equations of the constrained system are then obtained from: 


$$
\underline{\underline{M}} \underline{\ddot{x}}+\underline{\underline{C}} \underline{\dot{x}}+\underline{\underline{K}} \underline{x}=\lambda \underline{\operatorname{grad} f}(\underline{0}) \text { with } \underline{x}^{T} \cdot \underline{\operatorname{grad} f}(\underline{0})=0
$$

It is easy from Equation [11] to obtain explicitly the Lagrange multiplier as a function of the state variables, such as:

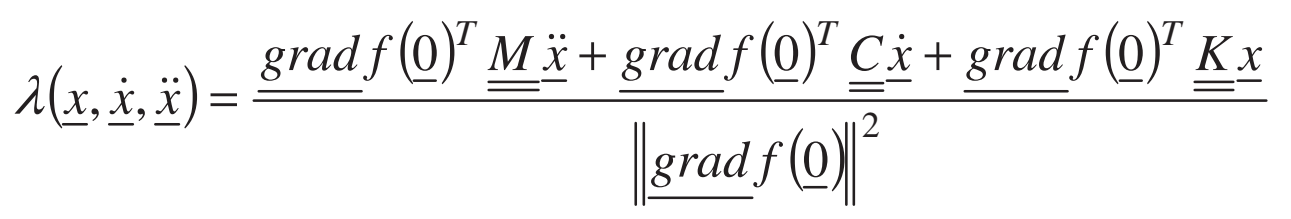

Moreover, some simplifications may occur for the undamped system $\underline{\underline{C}}=\underline{\underline{0}}$. The acceleration term can be obtained from Equation [11] by inverting the mass matrix:

$$
\underline{\ddot{x}}=\lambda \underline{\underline{M}}^{-1} \underline{\operatorname{grad}} f(\underline{0})-\underline{\underline{M}}^{-1} \underline{\underline{K}} \underline{x}
$$

The constraint Equation [11] can be also applied to the acceleration term:

$$
\underline{\ddot{x}}^{T} \cdot \underline{\operatorname{grad} f}(\underline{0})=0 \Rightarrow \lambda(\underline{x})=\frac{\underline{\operatorname{grad} f}(\underline{0})^{T} \underline{\underline{M}}^{-1} \underline{\underline{K}} \underline{x}}{\underline{\operatorname{grad} f(\underline{0})^{T} \underline{\underline{M}}} \underline{\underline{\operatorname{grad}} f(\underline{0})}}
$$

It should be mentioned that such a constraint system behaves like active controlled system, as recently studied by Nudehi et al. (2006) on the active vibration control of a column using a buckling-type force. Finally, the constraint undamped system can be written as:

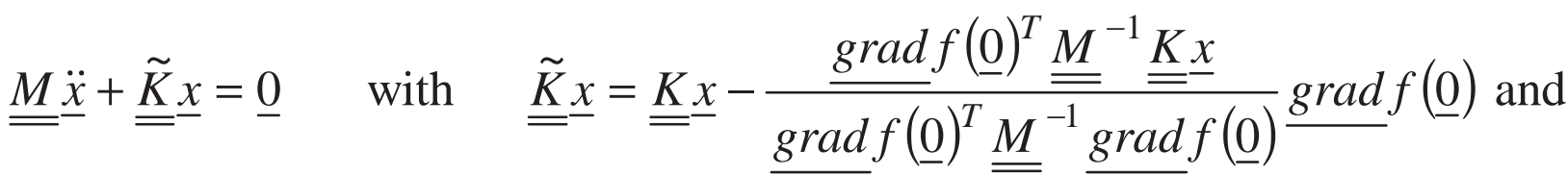

$$
\begin{aligned}
& \underline{x}^{T} \cdot \operatorname{grad} f(\underline{0})=0
\end{aligned}
$$

This is clearly a system of dimension (n-1), whose stability can be investigated from the minor of the stiffness matrix. There is no general theorem, to our knowledge, to investigate the stability of the constrained system from the stability of the free one. We suggest to study an academic structural example to illustrate the purpose.

\section{Example: non-conservative generalised Ziegler's column}

\subsection{Stability of the free system}

A simple non-conservative problem was chosen to illustrate the preliminary results, especially when the stiffness matrix $K$ is no longer symmetric. The Ziegler column, loaded by a partial follower load ( $\overline{\bar{H}}$ ermann and Bungay, 1964; Leipholz, 
1987) can be considered an interesting structural system, because instability by divergence and instability by flutter may both appear, depending on the structural parameters.

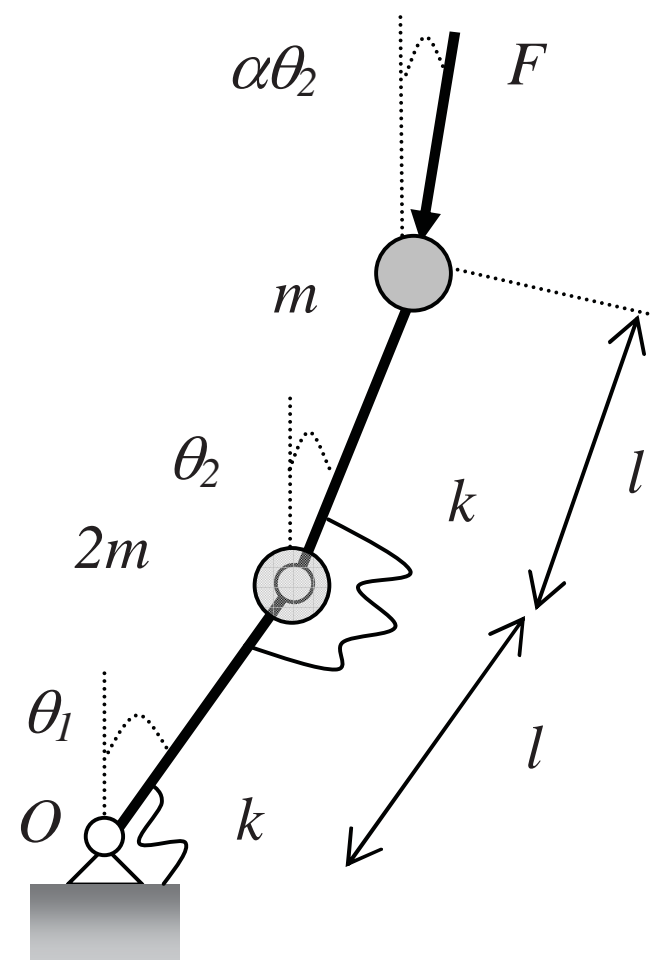

Figure 1. Generalised Ziegler's model subjected to partial follower load

This undamped structural system is sometimes called a generalised Ziegler column. It is a pinned column with a sub-tangential or super-tangential buckling load $F$ (sub-tangential for $\alpha<1$, super-tangential for $\alpha>1$ ). This is a two-degrees-offreedom system with a state vector $\underline{x}^{T}=\left(\theta_{1}, \theta_{2}\right)$, where $\theta_{i}$ is the rotation in each spring (see Figure 1). The stiffness of each spring is denoted by $k$. The dimensionless stiffness matrix is given by (see for instance Leipholz, 1987):

$$
\underline{\underline{K}}=\left(\begin{array}{cc}
2-p & \alpha p-1 \\
-1 & 1-(1-\alpha) p
\end{array}\right) \quad \text { and } \underline{\underline{M}}=m l^{2}\left(\begin{array}{ll}
3 & 1 \\
1 & 1
\end{array}\right) \quad \text { with } p=\frac{F l}{k}
$$

$p$ is the loading parameter and $\alpha$ is the parameter that characterises the orientation of the follower load (see Figure 1). $\alpha=0$ corresponds to the conservative case and $\alpha=1$ to the academic case of Ziegler's column. The "static" criterion can be applied to characterise the instability boundary by divergence (Equation [4]):

$$
\operatorname{det}(\underline{\underline{K}})=0 \Rightarrow(1-\alpha) p^{2}-3 p(1-\alpha)+1=0
$$

whereas the second-order work criterion Equation [6] leads to the lower bound: 


$$
\operatorname{det}\left(\underline{\underline{K}}^{S}\right)=0 \Rightarrow\left(1-\alpha-\frac{\alpha^{2}}{4}\right) p^{2}-3 p(1-\alpha)+1=0
$$

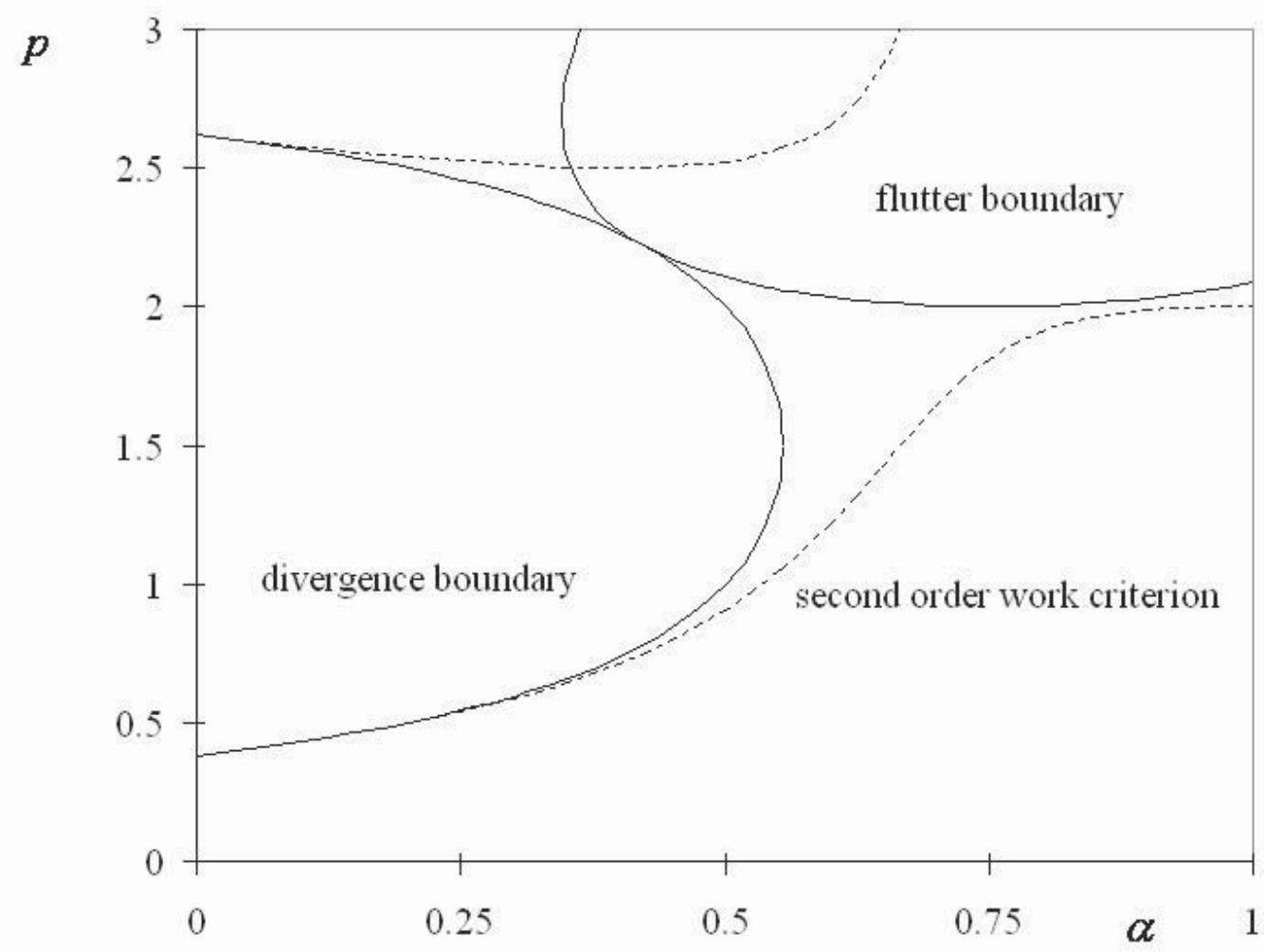

Figure 2. Stability diagram - Comparison with the second-order criterion

Obviously, both criteria coincide in the conservative example $(\alpha=0)$. It is easy to verify that Equation [18] is a lower bound of Equation [17] (see also Figure 2). We stress that no theoretical result can ensure the lower bound status of the secondorder criterion, when there is stability loss by flutter (even if this property is verified for this system). Flutter arises when one of the eigenvalues of the associated eigenvalue problem becomes complex with a positive real part. In the present case, the flutter load is calculated as (Hermann and Bungay, 1964; Leipholz, 1987):

$$
p=\frac{8-\alpha \pm \sqrt{(8-\alpha)^{2}-41\left(1+(1-\alpha)^{2}\right)}}{2\left[1+(1-\alpha)^{2}\right]}
$$

\subsection{Stability of the constraint system}

Ziegler's model under a partially follower load is considered again (Figure 1). Here, the factor control $\lambda$ is associated with the horizontal reaction load (this is similar to the reaction load considered by Kuznetsov and Levyakov (2002) for the Euler column). Following the notations of Equation [11] with $\underline{x}^{T}=\left(\theta_{1}, \theta_{2}\right)$, the additional constraint is chosen as: 


$$
\sum_{k=1}^{n=2} x_{k}=0
$$

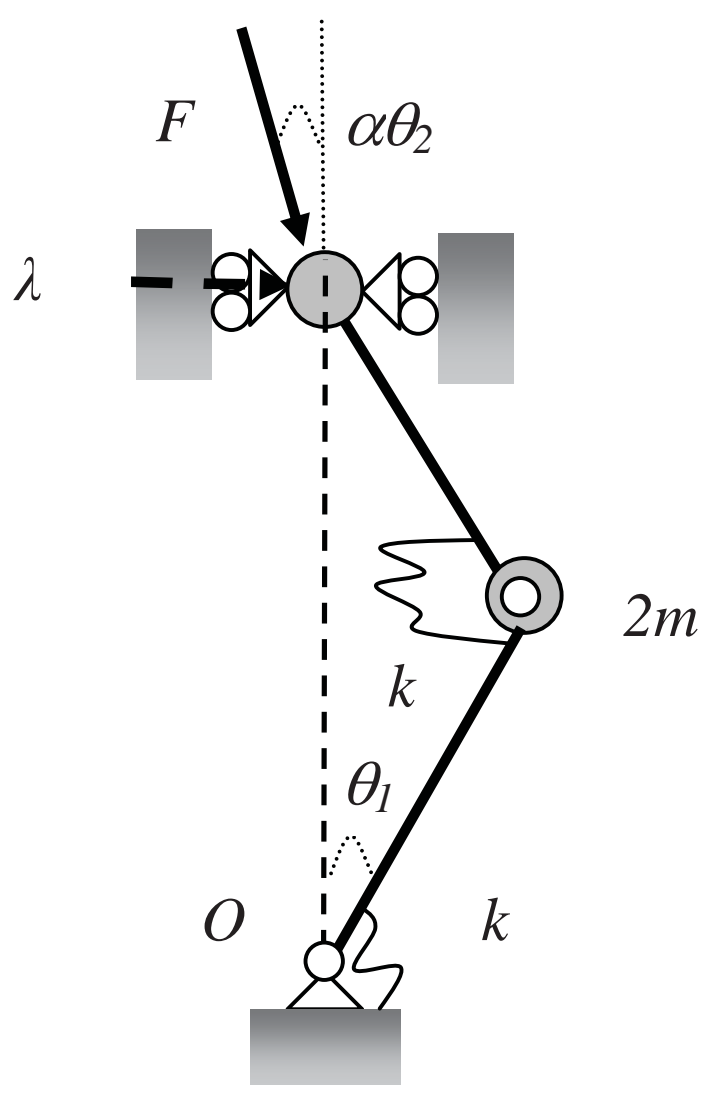

Figure 3. Generalised Ziegler's model subjected to partial follower load - Effect of an additional constraint $\lambda(t) / \theta_{1}(t)+\theta_{2}(t)=0$

This constraint can be understood as an isochoric loading. It is easy to check that the equilibrium configuration $\underline{x}=\underline{0}$ verifies this additional constraint. Effect of this additional constraint is illustrated in Figure 3. The new dynamical system is a singledegree-of-freedom system, and application of the divergence criterion (instability necessarily prevails by divergence for the single-degree-of-freedom system) to the system given by Equation [15] leads to:

$$
k_{11}+k_{22}-k_{12}-k_{21}=0
$$

where $\left(k_{i j}\right)$ are the terms of the stiffness matrix $\underline{\underline{K}}$. The instability load of the constrained system, (or isochoric system) is calculated as:

$$
\theta_{1}+\theta_{2}=0 \Rightarrow p=\frac{5}{2}
$$

This value, which does not depend on the non-conservativeness parameter $\alpha$, can be found again by applying the virtual work principle: 


$$
5 k \theta_{1} \delta \theta_{1}-2 F l \cos \left(\alpha \theta_{1}\right) \sin \left(\theta_{1}\right) \delta \theta_{1}=0
$$

which leads to the post-buckling branch (affected by the parameter $\alpha$ ):

$$
p=\frac{5}{2} \frac{\theta_{1}}{\cos \left(\alpha \theta_{1}\right) \sin \left(\theta_{1}\right)}
$$

The buckling load of Equation [22] is found again from Equation [24]. This buckling load $(p=5 / 2)$ is much higher than that of the free conservative system $(p=(3-\sqrt{5}) / 2)$. This property is discussed by Ziegler (1968) for conservative systems: a clamped-hinged column has a higher load than a clamped-free column (see also Milner, 1995). However, one has to keep in mind that increasing stiffness does not necessarily increase the buckling load in the general case (as shown for instance by Tarnai $(1980,2002)$ for conservative systems).

We provide a simple example of a non-conservative system, whose increase in stiffness does not necessarily lead to an increase in the instability load. Consider Ziegler's model under a partial follower load, where the stiffness of the upper rotational spring tends to an infinite value (whereas the stiffness of the lower rotational spring is kept at a constant value denoted by $k$ ). This system is equivalent to a single-degree-of-freedom system with $\theta_{1}$ equal to $\theta_{2}\left(\theta_{1}-\theta_{2}=0\right)$. A column of length $2 l$ is attached to a spring of stiffness $k$ and subjected to a partially follower load. Application of the virtual work principle leads to:

$$
k \theta_{1} \delta \theta_{1}-2 F l \cos \left(\alpha \theta_{1}\right) \sin \left(\theta_{1}\right) \delta \theta_{1}+2 F l \sin \left(\alpha \theta_{1}\right) \cos \left(\theta_{1}\right) \delta \theta_{1}=0
$$

which leads to the post-buckling branch (affected by the parameter $\alpha$ ):

$$
p=\frac{\theta_{1}}{2 \sin \left((1-\alpha) \theta_{1}\right)}
$$

Hence, the buckling load depends on the parameter $\alpha$, in this case, and is equal to:

$$
\theta_{1}-\theta_{2}=0 \Rightarrow p=\frac{1}{2(1-\alpha)}
$$

Figure 4 and Figure 5 show a comparison between the stability boundary of the free system (system of Figure 1), the constrained system with the incompressibility (or isochoric) condition $\left(\theta_{1}+\theta_{2}=0\right)$, and the constrained system that has one spring with infinite stiffness $\left(\theta_{1}-\theta_{2}=0\right)$. 


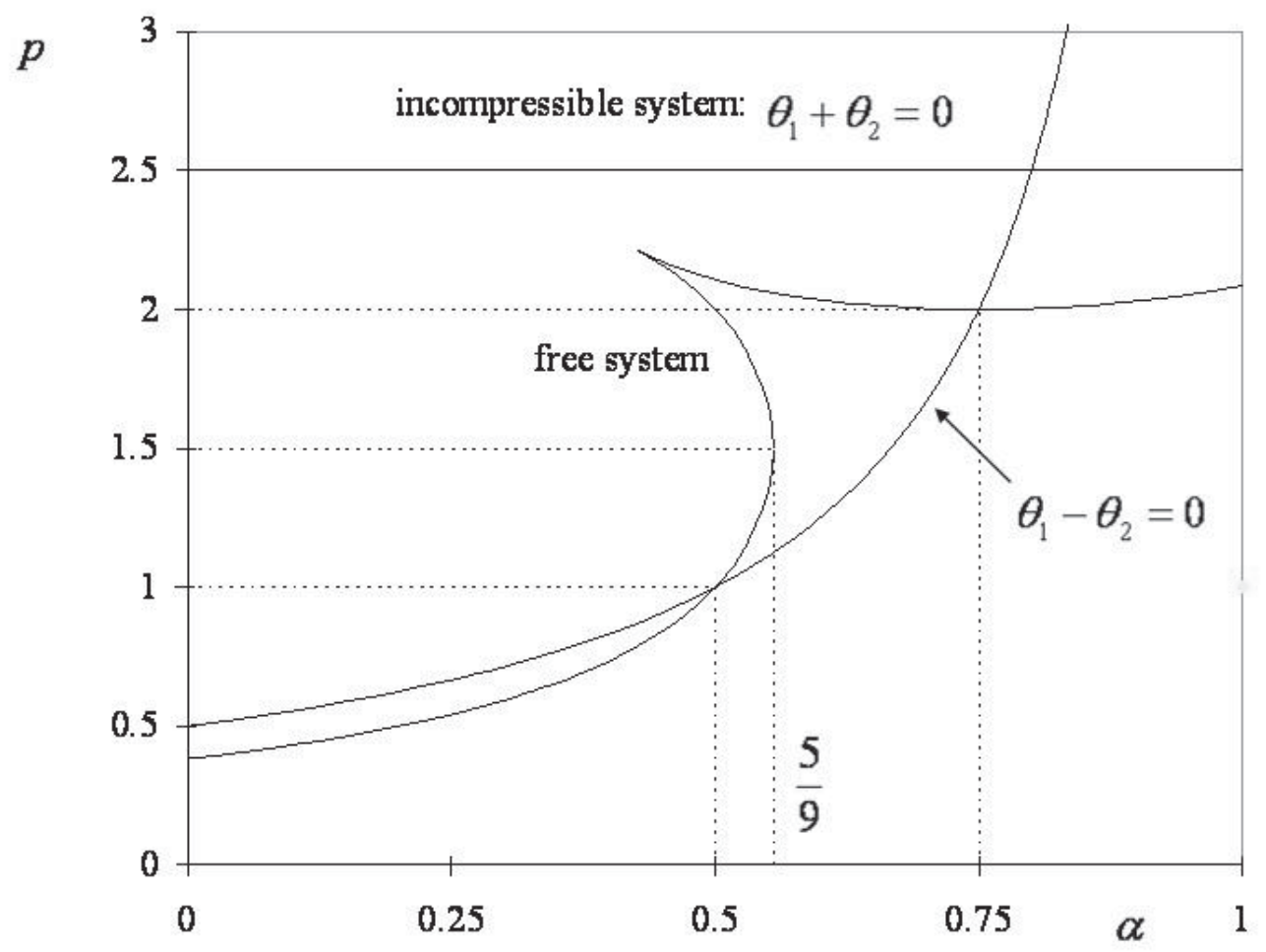

Figure 4. Comparison of the instability load of the free and the constrained system

It is observed that there is a region $\alpha \in[1 / 2 ; 3 / 4]$ where the system with an infinite stiffness has a lower instability load than the initial one, even in the divergence transition area $\alpha \in[1 / 2 ; 5 / 9]$. For this non-conservative system, an increase in stiffness may destabilise the system, even if only divergence instabilities are considered.

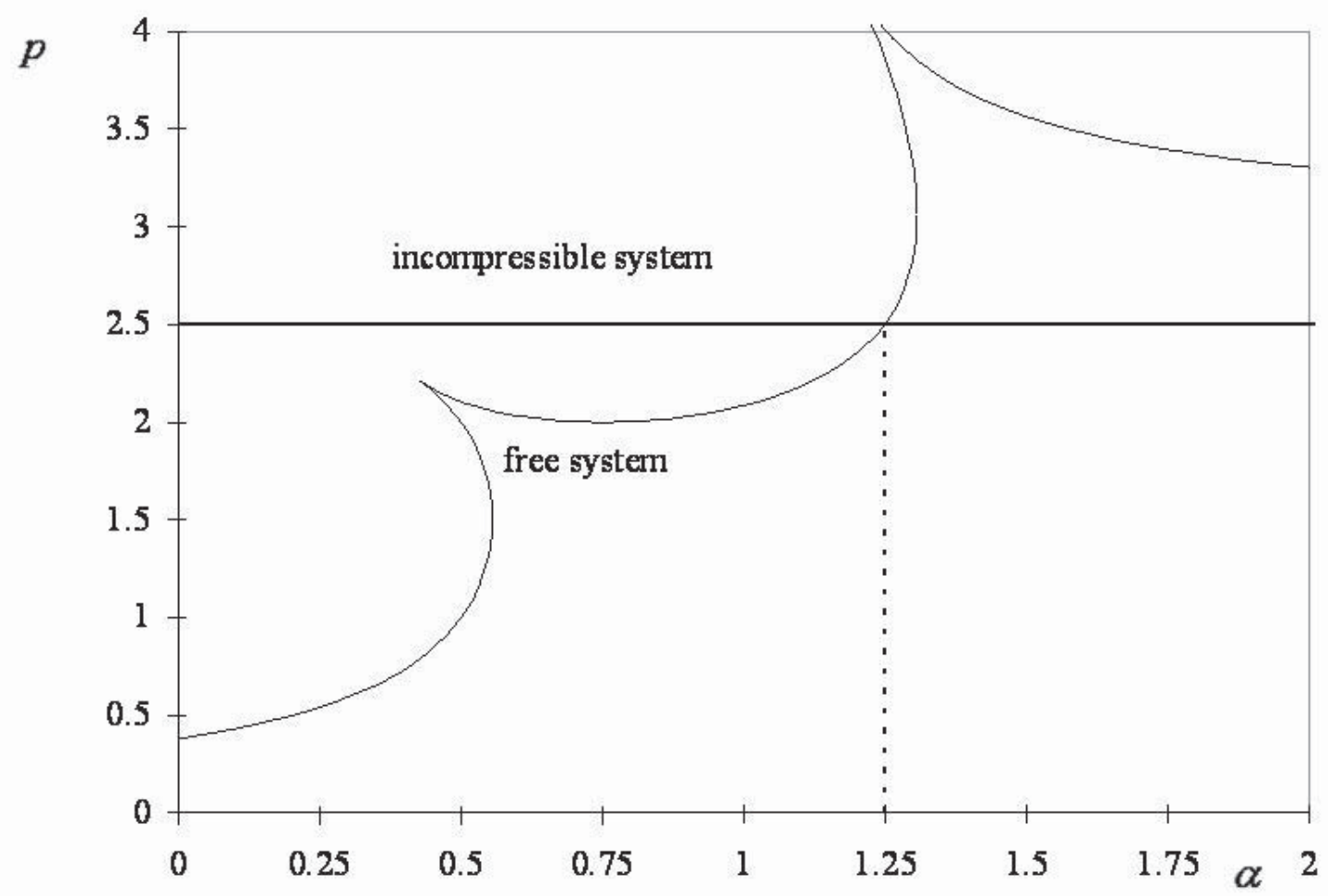

Figure 5. Effect of isochoric loading on stability boundary 
Finally, a displacement constrained case may be studied as in Figure 6.
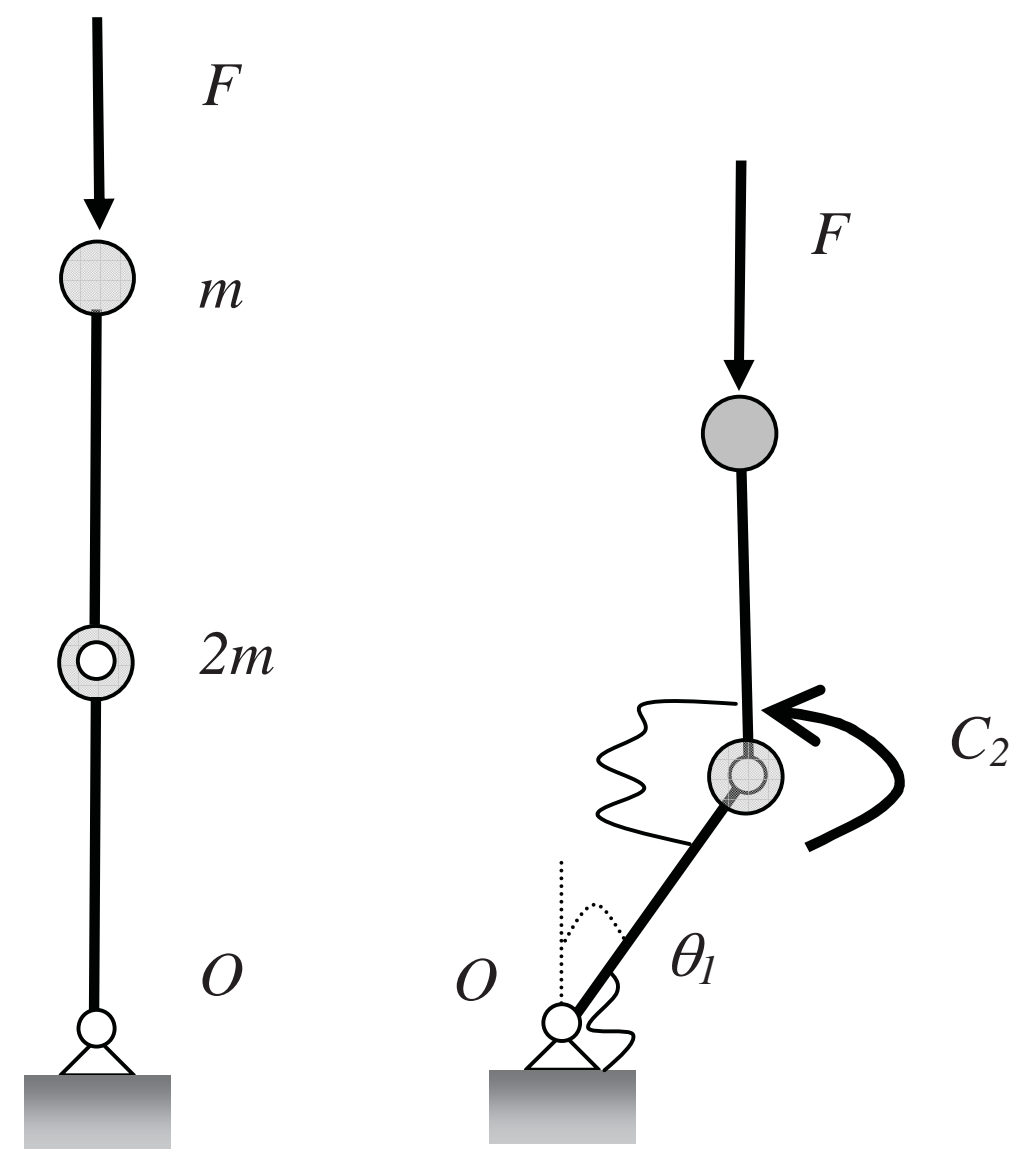

Figure 6. Stability of Ziegler's model with the constraint $\theta_{2}=0$

Suppose for instance that $\theta_{2}$ is vanishing $\left(\theta_{2}=0\right)$, as an additional constraint associated with a torque $C_{2}$, which can be considered as a Lagrange multiplier. In this case, the stability limit is obtained from application of Equation [15] with the constraint $\theta_{2}=0$ leading to:

$$
\theta_{2}=0 \Rightarrow k_{11}=0 \Rightarrow p=2
$$

\section{Continuum analogy}

\subsection{Beck's column}

The continuum analogy of Ziegler's column is Beck's column (clamped-free condition; Beck, 1952). It could be said that the continuum analogy of the generalised Ziegler problem under partial follower load is Contri's column (Contri, 1966). The effect of the discretisation process on the stability diagram has been studied in detail by Gasparini et al. (1995). For clamped-hinged conditions, the column stability problem under follower axial load has been investigated by Leipholz (1987), for example. The follower load column under clamped-hinged 
conditions has recently been analysed by Plaut (2006) in the post-buckling range. Plaut (2006) also showed the analogy with the problem of pipe conveying fluid. Indeed, Figure 7 can be understood as the continuum generalisation of the model presented in Figure 3.

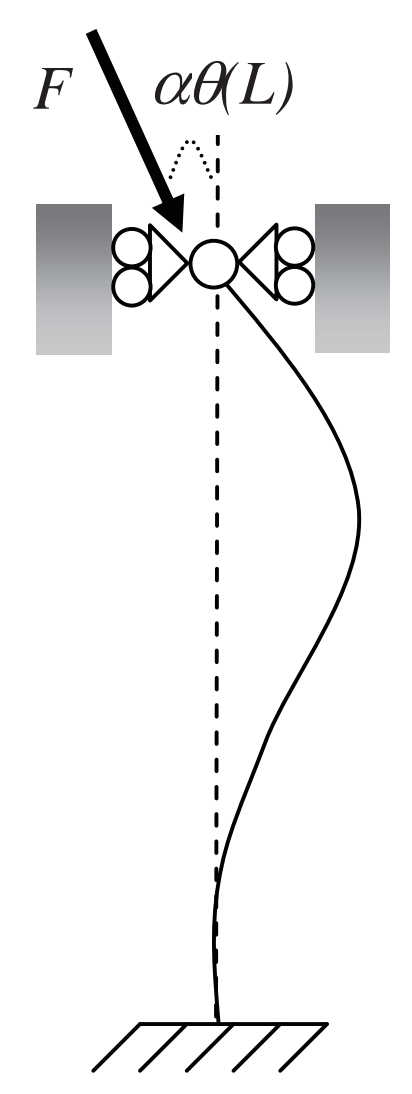

Figure 7. Concentrated follower load-a clamped-hinge condition

The continuum isochoric condition Equation [20] would be written as:

$$
\int_{0}^{L} \sin \theta(s) d s=0
$$

The buckling load of such a system is the same as the buckling load of the conservative system (with a vertical load). This result was found again for the discretised version of the continuum column, where the buckling load was found to be independent of $\alpha$ (see Equation [22]). However, as shown in the present paper (see Equation [24]), the post-buckling behaviour depends on the non-conservative parameter $\alpha$, and we can say that the system represented in Figure 7 is nonconservative in the large. Nevertheless, it is not within the scope of this paper to consider the overall displacement behaviour of this non-conservative problem (see (Plaut, 2006)), since the displacement behaviour of the conservative problem is already quite complex from a theoretical and numerical point of view (Wang, 1997; Mikata, 2007). 
These results would lead to the conclusion that the discretised and continuous column problem finally shows substantial similarities, except possibly the disappearance of the instability domain by divergence for large values of the nonconservative parameter $\alpha$ in the continuous column (Gasparini et al., 1995).

\subsection{Some implications in soil mechanics}

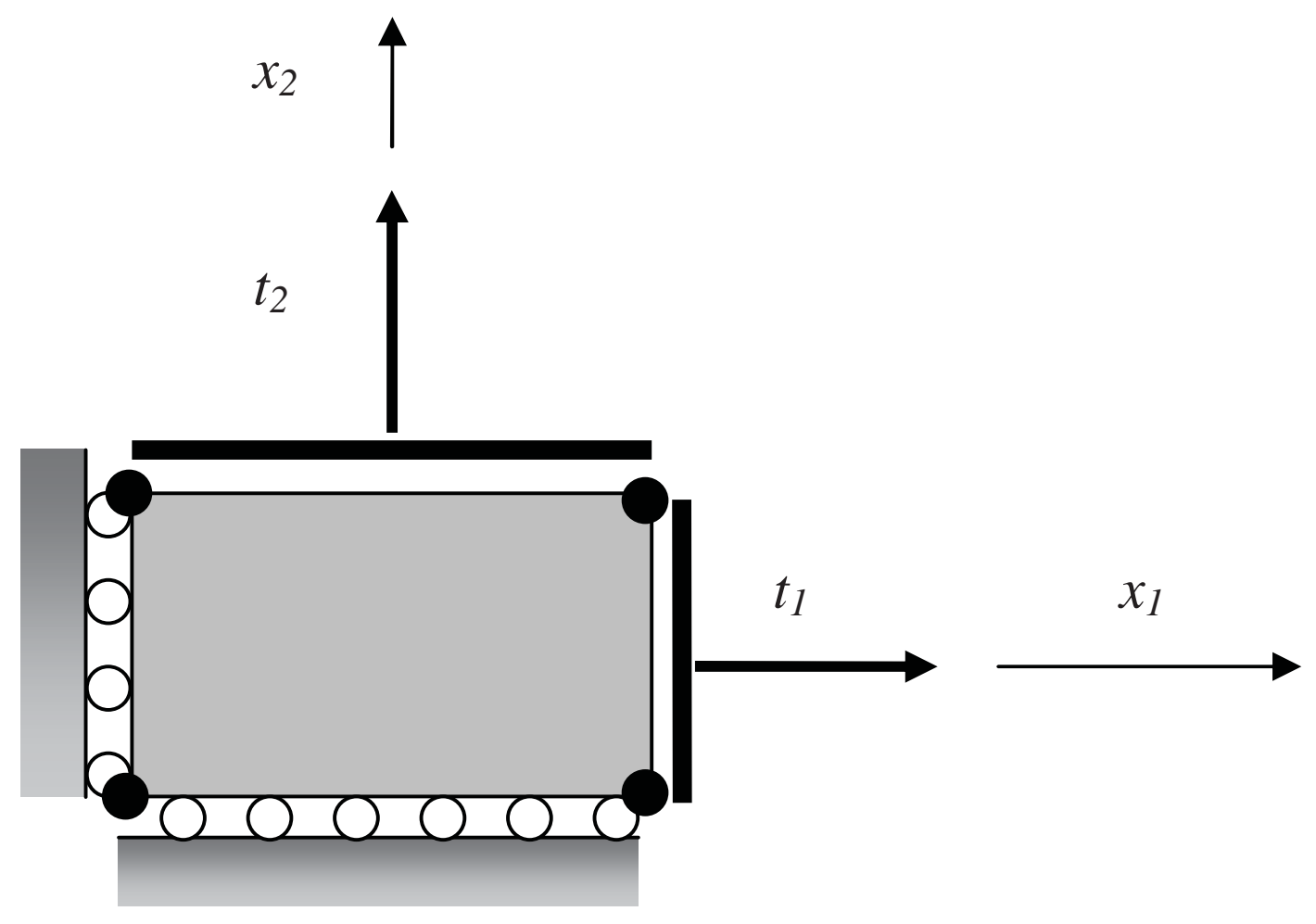

Figure 8. A two-degrees-of-freedom system with a non-conservative law

The stability of the discretised system depicted in Figure 8 is studied. This could be the discretised version of a Finite Element model to two degrees-of-freedom in plane strain conditions.

The first-order isochoric condition is written for a square element as:

$$
x_{1}+x_{2}=\underline{1}^{T} \underline{x}=0 \text { with } \underline{1}=\left(\begin{array}{l}
1 \\
1
\end{array}\right)
$$

One recognizes of course Equation [20]. The material tested is assumed to be linearly elastic and eventually non-conservative (a non-symmetric elastic stiffness matrix). Such constitutive relations can be encountered when modelling anisotropic damage in quasi-brittle materials with some unilateral effects (Carol and Willam, 1996; Challamel et al., 2006a). We do not discuss the realistic nature of such constitutive law, given that the non-conservativeness provided from the loading aspect already a controversial topic (Elishakoff, 2005; Challamel et al., 2009). The 
non-symmetric stiffness matrix can also be referred to the elasto-plastic behavior of a geomaterial with a non-associated flow rule, whose incremental form may lead to a non-symmetric stiffness matrix.

It is worth mentioning that the isochoric condition (see Equation [20]) does not necessarily lead to a safe upper bound of the stability boundary, as shown in Figure 5. It can be observed in Figure 5 that a critical parameter $\alpha$ exists where the incompressibility condition leads to a lower bound of the stability boundary $\left(\alpha_{c r} \cong 1.25\right)$. A comparison between this structural example and various instabilities found in soil mechanics may be interesting to investigate. In fact, the isochoric test of loose sand may decrease the instability boundary, whereas for a dense sand, the isochoric test may increase the instability boundary. In this formal analogy, the density parameter plays a role similar to that played by the non-conservativeness parameter $\alpha$ in the generalised version of Ziegler's model.

In static analysis, the force-displacement relation can be written as:

$$
\underline{\underline{K}} \underline{\underline{x}}=\underline{F}
$$

It can be relevant to introduce the control parameters:

$$
x_{v}=x_{1}+x_{2} \text { and } q=F_{1}-F_{2}
$$

Equation [31] can be decomposed in the matrix form:

$$
\left(\begin{array}{cc}
1 & -1 \\
0 & 1
\end{array}\right)\left(\begin{array}{l}
F_{1} \\
F_{2}
\end{array}\right)=\left(\begin{array}{cc}
1 & -1 \\
0 & 1
\end{array}\right)\left(\begin{array}{ll}
k_{11} & k_{12} \\
k_{21} & k_{22}
\end{array}\right)\left(\begin{array}{ll}
1 & 0 \\
1 & 1
\end{array}\right)^{-1}\left(\begin{array}{ll}
1 & 0 \\
1 & 1
\end{array}\right)\left(\begin{array}{l}
x_{1} \\
x_{2}
\end{array}\right)
$$

It can be shown that Equation [33] can be also written as:

$$
\left(\begin{array}{ll}
a & b \\
c & d
\end{array}\right)\left(\begin{array}{l}
x_{1} \\
x_{v}
\end{array}\right)=\left(\begin{array}{l}
q \\
F_{2}
\end{array}\right) \quad \text { with }\left\{\begin{array} { l } 
{ a = k _ { 1 1 } + k _ { 2 2 } - k _ { 1 2 } - k _ { 2 1 } } \\
{ b = k _ { 1 2 } - k _ { 2 2 } }
\end{array} \text { and } \left\{\begin{array}{l}
c=k_{21}-k_{22} \\
d=k_{22}
\end{array}\right.\right.
$$

Finally, the relationship between the control parameters and the state parameters can be expressed by:

$$
d^{-1}\left(\begin{array}{cc}
a d-b c & b \\
-c & 1
\end{array}\right)\left(\begin{array}{l}
x_{1} \\
F_{2}
\end{array}\right)=\left(\begin{array}{l}
q \\
x_{v}
\end{array}\right) \text { for } d \neq 0
$$

The invertibility condition is simply expressed when the determinant of the matrix introduced in Equation [35] does not vanish:

$$
a \neq 0 \quad \Leftrightarrow \quad k_{11}+k_{22}-k_{12}-k_{21} \neq 0
$$


This is also the controllability condition (in Nova's sense) to achieve a test with a prescribed value of $x_{v}$ (and in particular, the isochoric condition $x_{v}=0$ ). Equation [36] can be satisfactorily compared to Equation [21]. Moreover, it can be shown (Nicot et al., 2007) that the second-order work vanishes along the isochoric path, when Equation [21] is valid:

$$
W_{2}=x_{1} F_{1}+x_{2} F_{2}=\underline{x}^{T} \underline{\underline{K}} \underline{x}=\left(k_{11}+k_{22}-k_{12}-k_{21}\right) x_{1}^{2} \text { if } x_{1}+x_{2}=0
$$

However, the Equation [21] criterion is different from the second-order work criterion Equation [6], as clearly highlighted below:

$$
\operatorname{det}\left(\underline{\underline{K}}{ }^{S}\right)=k_{11} k_{22}-\left(\frac{k_{12}+k_{21}}{2}\right)^{2}=-\left(\frac{k_{11}-k_{22}}{2}\right)^{2} \leq 0 \text { if } k_{11}+k_{22}-k_{12}-k_{21}=0
$$

Equation [38] shows that the second-order work criterion Equation [6] constitutes a lower bound to the instability criterion associated with the isochoric loading (if instability prevails by divergence). In other words, when the second-order work vanishes along the isochoric path, the determinant of the symmetric part of the tangent stiffness matrix has already vanished.

It is worth mentioning that there exits other constrainsts where instability is reached before the instability load associated with the isochoric loading. This is for instance the case of a displacement constrained test with $x_{2}$ fixed, leading in this case to a much smaller instability load (see Equation [28]). Such a loading device is often considered in soil mechanics, in presence of mixed perturbations. In Equation [28], for instance, the criterion explicitly given by Nova (2004) with loaddisplacement controlled tests can be noted.

The isochoric condition can also be regarded as a particular case of the more general proportional kinematic condition: the lateral plates are displaced in such a way that strains increase in proportion to each other, with a given proportionality value $\lambda_{0}\left(x_{1}+\lambda_{0} x_{2}=0\right)$. In this case, the second-order work is vanishing for:

$$
W_{2}=x_{1} F_{1}+x_{2} F_{2}=\underline{x}^{T} \underline{\underline{K}} \underline{x}=\left(\lambda_{0}{ }^{2} k_{11}+k_{22}-\lambda_{0} k_{12}-\lambda_{0} k_{21}\right) x_{2}{ }^{2} \text { if } x_{1}+\lambda_{0} x_{2}=0
$$

Therefore, the stability boundary for this structural problem with mixed loading is obtained from:

$$
x_{1}+\lambda_{0} x_{2}=0 \Rightarrow \lambda_{0}^{2} k_{11}+k_{22}-\lambda_{0} k_{12}-\lambda_{0} k_{21}=0
$$


which is a generalization of Equation [21] for proportional kinematic conditions (this can be also found again from Equation [15]). The isochoric case corresponds to a proportionality value $\lambda_{0}$ equal to 1 . Furthemore,

$$
\begin{aligned}
\operatorname{det}\left(\underline{\underline{K^{S}}}\right)= & k_{11} k_{22}-\left(\frac{k_{12}+k_{21}}{2}\right)^{2}=-\left(\frac{k_{11} \lambda_{0}^{2}-k_{22}}{2 \lambda_{0}}\right)^{2} \leq 0 \text { if } \\
& \lambda_{0}{ }^{2} k_{11}+k_{22}-\lambda_{0} k_{12}-\lambda_{0} k_{21}=0
\end{aligned}
$$

Hence, excluding flutter instabilities, the condition $\operatorname{det}\left(\underline{\underline{K^{S}}}\right)=0$ is a sufficient condition of stability (lower bound of the stability boundary of the free system, and lower bound of the stability boundary of the system for proportional kinematic conditions). Applying the criterion Equation [40] leads to the stability boundary of the system for proportional kinematic conditions, parameterised by $\lambda_{0}$ as:

$$
p=\frac{2 \lambda_{0}^{2}+2 \lambda_{0}+1}{\lambda_{0}^{2}+\lambda_{0} \alpha+1-\alpha}
$$

The smallest instability load is obtained for:

$$
\frac{\partial p}{\partial \lambda_{0}}=0 \Rightarrow \lambda_{0}=\frac{2 \alpha-1+\sqrt{(2 \alpha-1)^{2}-(2 \alpha-2)(2-3 \alpha)}}{2(\alpha-1)}
$$

It can be shown that this characteristic value injected in the parametric stability boundary Equation [42] leads to the second-order work criterion $\operatorname{det}\left(\underline{\underline{K^{S}}}\right)=0$, given by Equation [18]. In fact, as shown by Nicot et al. (2008), when the condition $\operatorname{det}\left(\underline{\underline{K^{S}}}\right)=0$ is met, the second order work vanishes along a given kinematic direction corresponding to a certain proportionality value generally different to 1 . For the present problem, this characteristic proportionality value is calculated from Equation [41] as:

$$
\lambda_{0}^{2}=\frac{k_{22}}{k_{11}}=\frac{1-(1-\alpha) p}{2-p}
$$

Equation [43] and Equation [44] are equivalent when the stability value of the parameter $p$ is introduced from Equation [42]. As a consequence, excluding flutter instabilities, the condition $\operatorname{det}(\underline{\underline{K}})=0$ is not only a lower bound of the stability boundary of the free system, but also the boundary of the stability domain, for all mixed perturbations based on proportional kinematic conditions. 


\section{Conclusions}

In conclusion, the results presented may be summarised as follows. For conservative systems, the stability criterion is given by the vanishing of the determinant of the stiffness matrix $\left(\operatorname{det}(\underline{\underline{K}})=\operatorname{det}\left(\underline{\underline{K}}{ }^{S}\right)=0\right)$. For non-conservative elastic systems, the static criterion also holds in cases of divergence instabilities $(\operatorname{det}(\underline{K})=0)$. For this type of instability, the second-order work criterion $(\operatorname{det}(\underline{\underline{K}} S)=0)$ constitutes a lower bound of the stability boundary (Huseyin and Leipholz, 1973). In cases of flutter instability, no theorem guarantees the lower bound status of the second-order work criterion. In other words, only flutter instabilities can precede the second-order work criterion.

Incompressible solids (or solids under isochoric loading) were also studied in this paper. Material incompressibility may arise for inelastic media, but structural incompressibility is often encountered in structural mechanics, when constraints are added at the structural level. For the constrained system (with isochoric loading), the constraint is a state control law which also depends on the mass matrix. Incompressibility strongly affects the instability criterion, which is detailed for a twodegrees-of-freedom system. A specific example shows that incompressibility increases the buckling load for conservative problems (as observed by Ziegler, 1968). For the non-conservative problem, the incompressibility condition does not necessarily give an upper bound to the free problem. In the present example, this depends on the degree of non-conservativeness (measured by the parameter $\alpha$ ). We keep in mind that in some cases an increase in stiffness in a structure may also decrease the buckling load, even for conservative systems (Parnes, 1977; Tarnai, 1981; see also Tarnai, 2002). This phenomenon was also observed in non-conservative systems controlled by the flutter phenomenon (Sundararajan, 1974). We give another example of this phenomenon from Ziegler's column under partial follower load. In the general case (and specifically, at the material scale), it was not possible to classify both stability domains (free problem and constrained problem) systematically, and a specific computation of the stability domains was therefore necessary.

Excluding flutter instabilities, the second-order work criterion is not only a lower bound of the stability boundary of the free system, but also the boundary of the stability domain, for all mixed perturbations based on proportional kinematic conditions. The conclusions of this paper, and in particular the instability nature by divergence maybe strongly affected by infinitesimal damping (see for instance Kounadis, 2007).

\section{References}

Absi E., Lerbet J., "Instability of elastic bodies", Mechanics Research Communications, vol. $31, \mathrm{n}^{\circ} 1,2004$, p. 39-44. 
Afolabi D., "Sylvester's eliminant and stability criteria for gyroscopic systems", Journal of Sound and Vibration, vol. 182, $\mathrm{n}^{\circ} 2,1995$, p. 229-244.

Bažant Z.P., Cedolin L., Stability of structures - Elastic, Inelastic, Fracture, and Damage Theories, Dover Publications, Inc., New York, 2003.

Beck M., "Die Knicklast des einseitig eingespannten tangential gedrückten Stabes", Zeitschrift für Angewandte Mathematik und Physik, vol. 3, 1952, p. 225-228, p. 476-477.

Bolotin V.V., Nonconservative problems of the theory of elastic stability, New York, Pergamon Press, 1963.

Carol I., Willam K., "Spurious energy dissipation/generation in stiffness recovery models for elastic degradation and damage", International Journal of Solids and Structures, vol. 33, $\mathrm{n}^{\circ} 20-22,1996$, p. 2939-2957.

Challamel N., Comments on the paper "Instability of elastic bodies" written by E. Absi and J. Lerbet (Mech. Res. Comm., vol. 31, $\mathrm{n}^{\circ}$ 1, 2004, p. 39-44), Mechanics Research Communications, vol. 32, 2005, p. 610-612.

Challamel N., Casandjian C., Lerbet J., "On the occurrence of flutter in the lateral-torsional instabilities of circular arches”, Journal of Sound and Vibration, 2009.

Challamel N., Gilles G., "Stability and dynamics of a harmonically excited elastic-perfectly plastic oscillator", Journal of Sound and Vibration, vol. 301, 2007, p. 608-634.

Challamel N., Halm D., Dragon A., "On the non-conservativeness of a class of anisotropic damage models with unilateral effects", Compte-Rendu de Mécanique, vol. 334, $\mathrm{n}^{\circ} 7$, 2006a, p. 414-418.

Challamel N., Lanos C., Casandjian C., "Stability analysis of quasi-brittle materials - creep under multiaxial loading", Mechanics of Time-dependent Materials, vol. 10, $\mathrm{n}^{\circ}$ 1, 2006b, p. 35-50.

Challamel N., Pijaudier-Cabot G., "Stability and dynamics of a plastic softening oscillator", Int. J. Solids Structures, vol. 43, 2006c, p. 5867-5885.

Chambon R., Caillerie D., Viggiani G., "Loss of uniqueness and bifurcation versus instability: some remarks", Revue française de génie civil, vol. 8, n 5-6, 2004, p. 517-535.

Contri L., "On the stability of elastic system under non-conservative follower forces", Meccanica, vol. 1, $\mathrm{n}^{\circ} 1-2,1966$, p. 61-64.

Darve F., Laouafa F., "Instabilities in granular materials and application to landslides", Mechanics of Cohesive-Frictional Materials, vol. 5, 2000, p. 627-652.

Darve F., Servant G., Laouafa F., Khoa H.D.V., "Failure in geomaterials, Continuous and discrete analyses", Computer Methods in Applied Mechanics and Engineering, vol. 193, $\mathrm{n}^{\circ}$ 27-29, 2004, p. 3057-3085.

Darve F., Vardoulakis I., Instabilities and Degradations in Geomaterials, Darve F., Vardoulakis I. (Eds), CISM Course, Springer, Berlin, 2005.

Elishakoff I., "Controversy associated with the so-called "follower forces": critical overview", Applied Mechanics Reviews, vol. 58, n 1-6, 2005, p. 117-142. 
Gallina P., "About the stability of non-conservative undamped systems", Journal of Sound and Vibration, vol. 262, 2003, p. 977-988.

Gajewski A., Zyczkowski M., Optimal structural design under stability constraints, Kluwer Academic Publishers, Dordrecht, The Netherlands, 1988.

Gasparini A.M., Saetta A.V., Vitaliani R.V., "On the stability and instability regions of nonconservative continuous system under partially follower forces", Computer Methods in Applied Mechanics and Engineering, vol. 124, 1995, p. 63-78.

Green A.E., Naghdi P.M., "On thermodynamics, rate of work and energy", Archive for Rational Mechanics and Analysis, vol. 40, 1971, p. 37-49.

Hermann G., Bungay R.W., "On the stability of elastic systems subjected to nonconservative forces", Journal of Applied Mechanics, vol. 86, 1964, p. 435-440.

Houlsby G.T., Amrosi A., Rojas E., "Elastic moduli of soils dependent on pressure: a hyperelastic formulation", Géotechnique, vol. 55, n 5, 2005, p. 383-392.

Huseyin K., Leipholz H., "Divergence instability of multiple-parameter circulatory systems", Quarterly Applied Mathematics, July, 1973, p. 185-197.

Kuznetsov V.V., Levyakov S.V., "Complete solution of the stability problem for elastica of Euler's column”, International Journal of Non-linear Mechanics, vol. 37, 2002, p. 1003-1009.

Imposimato S., Nova R., "An investigation on the uniqueness of the incremental response of elastoplastic models for virgin sand", Mechanics of Cohesive-Frictional Materials, vol. 3, 1998, p. 65-87.

Kounadis A.N., "Flutter instability and other singularity phenomena in symmetric systems via combination of mass distribution and weak damping", Int. J. Non-linear Mech., vol. 42, 2007, p. 24-35.

La Salle J., Lefschetz S., Stability by Liapunov's direct method with applications, Academic Press, New York, 1961.

Leipholz H., Stability theory, New York, John Wiley, 1987.

Lerbet J., Absi E., Rigolot A., "About the stability of nonconservative undamped elastic systems: some new elements”, Int. J. Struct. Stability Dynamics, 2009.

Mikata Y., "Complete solution of elastica for a clamped-hinged beam, and its applications to a carbon nanotube", Acta Mechanica, vol. 190, 2007, p. 133-150.

Milner H.R., "Stability of discrete systems", Summation Theorems in Structural Stability (CISM Courses n 354), T. Tarnai (Ed.), Springer-Verlag, Wien, 39-109, 1995.

Nguyen Q.S., Stabilité et mécanique non linéaire, Hermès, 2000.

Nicot F., Darve F., Khoa H.D.V., "Bifurcation and second-order work in geomaterial", International Journal of Numerical and Analytical Methods in Geomechanics, vol. 31, $\mathrm{n}^{\circ} 8,2007$, p. 1007-1032.

Nicot F., Sibille L., Darve F., "Bifurcation in granular materials: an attempt at a unified framework", International Journal of Solids and Structures. 
Nova R., "Controllability of geotechnical testing”, Revue française de génie civil, vol. 8, $\mathrm{n}^{\circ} 5 / 6,2005$, p. 613-634.

Nudehi S., Mukherjee R., Shaw S.W., "Active vibration control of a flexible beam using a buckling-type end force", Journal of Dynamic Systems, Measurement and Control ASME, vol. 128, 2006, p. 278-286.

Ostrowski A., Taussky O., "On the variation of the determinant of a positive definite matrix", Proceedings of the Koninklijke Nederlandse Academie van Wetenschappen, Series A, Mathematical Sciences, vol. 54, 1951, p. 383-385.

Parnes R., "A paradoxical case in a stability analysis", American Institute of Aeronautics and Astronautics Journal, vol. 15, 1977, p. 1533-1534.

Plaut R.H., "Postbuckling and vibration of end-supported elastica pipes conveying fluid and columns under follower loads", Journal of Sound and Vibration, vol. 289, 2006, p. 264-277.

Plaut R.H., "Snap loads and torsional oscillations of the original Tacoma Narrows bridges", Journal of Sound and Vibration, vol. 309, n 3-5, 2008, p. 613-636.

Plaut R.H., Davis F.M., "Sudden lateral asymmetry and torsional oscillations of section models of suspension bridges", Journal of Sound and Vibration, vol. 307, n 3-5, 2007, p. 894-905.

Rubin M.B., "On evolution equations for elastic deformation and the notion of hyperelasticity”, International Journal of Engineering Sciences, vol. 47, 2009, p. 76-82.

Sundararajan C., "Influence of end supports on the stability of nonconservative elastic systems", Journal of Applied Mechanics, vol. 41, 1974, p. 313-315.

Tarnai T., "Destabilizing effect of additional restraint on elastic bar structures", International Journal of Mechanical Sciences, vol. 22, 1980, p. 379-390.

Tarnai T., "Additional restraints can reduce the critical buckling loads and the natural frequencies", Proc. of the Second Int. Conf. on Structural Stability and Dynamics (Ed. C.M. Wang, G.R. Liu, K.K. Ang), World Scientific, Singapore, 2002, p. 88-97.

Tarnai T., "Paradoxical behaviour of vibrating systems challenging Rayleigh's theorem", $21^{\text {st }}$ International Congress of Theoretical and Applied Mechanics, Warsaw, 2004.

Wang C.Y., "Post-buckling of a clamped-simply supported elastica", International Journal of Non-linear Mechanics, vol. 32, n 6, 1997, p. 1115-1122.

Willam K.J., Iordache M.M., "On the lack of symmetry in materials", International Conference on Trends in Computational Structural Mechanics, Schloss Hofen, Austria, May 20-23, 2001, p. 233-242.

Ziegler H., Principles of Structural Stability, Blaisdell Publishing Company, 1968.

Zytynski M., Randolph M.F., Nova R., Wroth C.P., “On modelling the unloading-reloading behaviour of soils", Int. J. Num. Anal. Meth. Geomech., vol. 2, 1978, p. 87-93. 